\title{
A holistic approach to supporting distance learning using the Internet: transformation, not translation
}

\author{
Pete Thomas, Linda Carswell, Blaine Price, Marian Petre \\ Department of Computing, Open University, Milton Keynes, U.K. \\ Contact: p.g.thomas@open.ac.uk
}

Focus: holistic integration; transformation; scalability; student support

\begin{abstract}
This is the first of a series of papers describing how the Open University, a large distance education institution, has embraced the Internet as a medium for supporting its students, tutors, academics, and administrators throughout the education process. This paper reports on a holistic approach to integrating technology into the teaching process which addresses how to provide necessary functions in effective forms some traditional, some new. It describes the development and structure of actual systems, which incorporate electronic student registration; electronic assignment submission, marking, and recording; electronic tutorials and other interaction; and electronic examinations. It describes and summarizes some of the findings of the evaluation of those systems, including student, academic, and administrator feedback. Finally, this paper reflects on the benefits of an integrated holistic approach in exploiting the Internet's potential to support distance learning on a massive scale. Subsequent papers in this series will address specific areas in detail: the student experience, the role of the tutor in an electronic environment, and the institutional perspective.
\end{abstract}

\section{Introduction: recommending a holistic approach}

Universities respond to changes in society, and it is natural they should follow the trend to use technology (Adamson-Macedo, 1996). Educators are looking to technology to solve many of their problems; many universities are considering distance teaching as an economical solution to increased demands, and view the Internet as an appropriate support tool. The hope is that Internet-based teaching will allow educators to accommodate more students with diminishing funds - and at the same time allow them to improve their teaching to provide a better student experience (e.g., Reinhardt, 1995; Skillicorn, 1996). But why, so far, has the promise of many small attempts at innovation rarely been realized on a larger scale? We suggest that successful, large-scale Internet-based teaching relies on a holistic approach which encompasses:

- a culture of supported learning;

- recognition of costs;

- integration of technology with the administrative infrastructure, as well as with teaching practice;

- transformation of practices (both teaching and administrative) to take advantage of technology in order to provide needed functions, rather than superficial translation of existing practices. 
supported learning:

Many institutions are converting lecture notes or other paper-based materials to HyperText Markup Language (HTML) for the World-Wide Web, but, with little support provided for the student, the gains are minimal. Simply converting the publication medium is inadequate for supported distance education, which aims to engage the student in a 'community of learning'. Making sense of at-best-looselystructured resources on the Web - a rich environment, but one minimalistic in terms of scaffolding for concept and skill development — demands additional, unaccustomed skills from students (Laurillard, 1993). Instead, Internet-based teaching must provide a thorough and insightful support structure for largely independent study.

\section{hidden costs:}

Well-intentioned efforts have been known to replace existing effective methods with 'high-tech' versions that simply increase administrative costs without improving the system — and sometimes damaging it (Pilgrim and Leung, 1996). Technology has hidden costs, including increased (possibly different) administration to support the technology, demand for new backup systems in the adopted technology, and requirement for user support. Sometimes the outcomes do not warrant the investment. Only a holistic analysis will reveal all the costs of a technology.

integration with administrative infrastructure:

Often, technological innovations, while interesting and appealing, are isolated, addressing one component of a course in isolation from the rest of the curriculum, or addressing one course in isolation from the rest of the university system. Yet each technological innovation has knock-on effects, especially if it increases demands on staff time, increases equipment requirements for students, and complicates administration. Scaling up and acceptance depend on smooth interfaces between innovative and incumbent systems.

transformation:

Effective integration of new technology requires an understanding of the whole education process and a critical examination of its functions. On one hand, the innovation must be integrated with existing practice. On the other, we must seek to improve practice rather than simply translate it, if we hope to alleviate demands on staff and make improvements for students. What the popular enthusiasm for the Internet and superficial translation exercises tend to overlook is the fundamental question: whether techology's effect on the learning it is meant to support is constructive, rather than obstructive.

This rest of this paper gives an overview of our attempts to provide a robust and scaleable electronic teaching model that integrates Internet technology into all phases of the education process, from registration to examination. It describes the systems that are in place. The results of a large-scale evaluation of those systems, and analyses of their impact on students, academics, and administration, will be presented in the subsequent papers in this series. 


\section{Internet presentation trials in Computing}

We began a sequence of research and development trials in 1995 to address a range of technical challenges:

- How could students be registered electronically?

- How could assignments be captured as electronic documents?

- How could assignment be marked electronically?

- How could face-to-face tutorials be replaced by electronic ones?

- How could examinations be conducted electronically?

- How could an electronic system be scaleable, robust, and economical?

The trials required the development of a software infrastructure, adaptation of administrative procedures, and new teaching practices, all of which will be reported in this series of papers. This first paper presents an overview of the electronic system.

The first trial, in 1995 (Thomas et al, 1996), was essentially a feasibility study, involving 30 international students studying an introductory Computing course with two UK-based "tutors" (instructors who provide individual academic support for up to 25 students). The second, in 1996, was a large-scale trial on two courses (the introductory Computing course and an upper-level course on programming languages), involving some 250 students and 22 tutors in the Internet presentation. Both the Internet presentation and a cohort from the conventional presentation were evaluated, using data collected through questionnaires, interviews, day-long tutor debriefings, student feedback, interaction logs completed by tutors, electronic communications, assignments, and examination results.

\section{Background: supported distance education, ' $O U$-style'}

There are many models of distance teaching, varying with culture, scale, and operational procedures (Jones, 1996). Many are variations on campus-based teaching: allowing external students to study conventional courses part-time, or allowing students and instructor to communicate from different locations, or making conventional materials available outside the conventional course.

Many are "same time, any place" models, which provide some freedom of location within conventional, campus-based teaching. Many examples in North America (Owen, 1996), have students receiving conventional lectures via satellite or video link. Students are physically removed from the instructor, but the educational experience is the same as for conventional students on campus. In other examples, small groups of students, (typically campus-based), are encouraged to submit work electronically to their instructor for it to be marked on screen (Pitt, 1996).

Other models, often of limited scope, make traditional learning materials available electronically as self-study materials. However, such materials were not originally intended for remote study, and they fail to address the particular needs of the distance learner.

In contrast, the Open University's supported open learning model of distance education is "any time, any place" and provides a network of student support systems and resources specifically designed for the distance learner, including specially- 
designed self-study texts and recordings, broadcast lectures, home experiment kits that 'turn a kitchen into a laboratory', educational software, regional face-to-face tutorial sessions, a personal (usually local) tutor who marks assignments and provides academic support, short residential schools, counselling, and self-help groups. The aim is to provide a mode of flexible study specifically for the distance learner who may be isolated by location or other circumstances, and to do so in a way that both maintains quality of education and "open and equal" access to education for all who desire it. The Open University has no admissions requirements; a 'typical' OU student is a mature student studying part-time at home, in full-time employment, and having family responsibilities.

The course materials have a dual role: to provide all necessary study information, and to foster self-study skills. The mainstay of teaching is the tutor-marked assignment (TMA). Throughout the course, the student is required to submit written assignments, which the tutor grades and annotates freely, providing essential feedback. The TMA has two roles: summative and formative, for assessing student progress and as a teaching tool for taking remedial action. In the conventional system, tutors support students by providing one-to-one interactions via post or telephone, and by offering short face-to-face teaching sessions or 'tutorials'. Students may also choose to form 'self-help groups' with other students.

This model has been fine-tuned over more than 25 years to provide high-quality university education to some 150,000 part-time students studying at a distance. The typical retention rate (those taking the examination) is in excess of $70 \%$, of whom $95 \%$ pass. It is a secure, robust, large-scale operation. To ensure quality, both the administrative system and the course production system consist of standardised procedures and a host of monitoring and regulating systems, making change difficult other than on an institutional level. An electronic alternative must prove its viability and functionality before such institutional changes are possible.

\section{Moving to Internet presentation}

The key challenges for a large, distance teaching university are scale and rapid feedback to a dispersed student population.

Scale:

In some courses in the Open University, there are as many as 8,000 students, and the total number of current students exceeds 150,000.

\section{Rapid feedback:}

Approximately some 40,000 assignments are processed per week, the efficiency of the U.K. postal service means that the students usually receive their marked assignments within a week (often within days) of the tutor marking and returning them. Retaining and motivating students hinges on rapid feedback, which makes communication (paper-based or otherwise) a key issue. This has been one of the barriers to the OU teaching outside the UK, where communication systems are often less reliable. Even within the U.K., despite the attention given to student support, students can still be disadvantaged: access to phone, fax, or face-to-face interaction may be restricted by a variety of circumstances. As a result, many students rely solely on the printed materials and graded assignment correspondence with their tutor. 
These are impairments to the "any time, any place" model and are obstacles in supporting students further afield than Continental Western Europe.

The potential afforded by electronic communication prompted thorough analysis of the four corners of the OU system:

- registration and the general administrative process

- assignment handling: submission, marking, return, and monitoring of assignments

- student-tutor interaction: queries and tutorials

- the examination process.

Any solution must observe the OU ethos of being "open and equal" with respect to technology access, both in the UK and abroad. Hence the backbone of our technology is plain-text Internet e-mail, which can cater for almost any student, regardless of the speed of the network connection or software available. It is hoped that electronic communication will have significant impact in a variety of areas: overcoming access barriers, providing flexibility and responsiveness in accommodating various student circumstances, providing automatic records and checks against minor errors, and providing efficient tools for administrators and overstretched tutors. However, an electronic system must prove itself secure, robust, scalable, and affordable. Each of the electronic systems will be examined in turn.

\section{Registration system}

Students enrol each year for their intended course of study. This is a two stage process: first, students reserve a place on their selected course(s) and receive course information; second, they register for their chosen course(s). In the paper-based registration process, students write, phone, or fax the University. All student information is captured on paper and entered manually into a database. If students are within the same country or time zone this sort of access is not problematic, but, for students who are geographically remote, postal delays, telephone costs, and differences in time zone can become obstacles. 


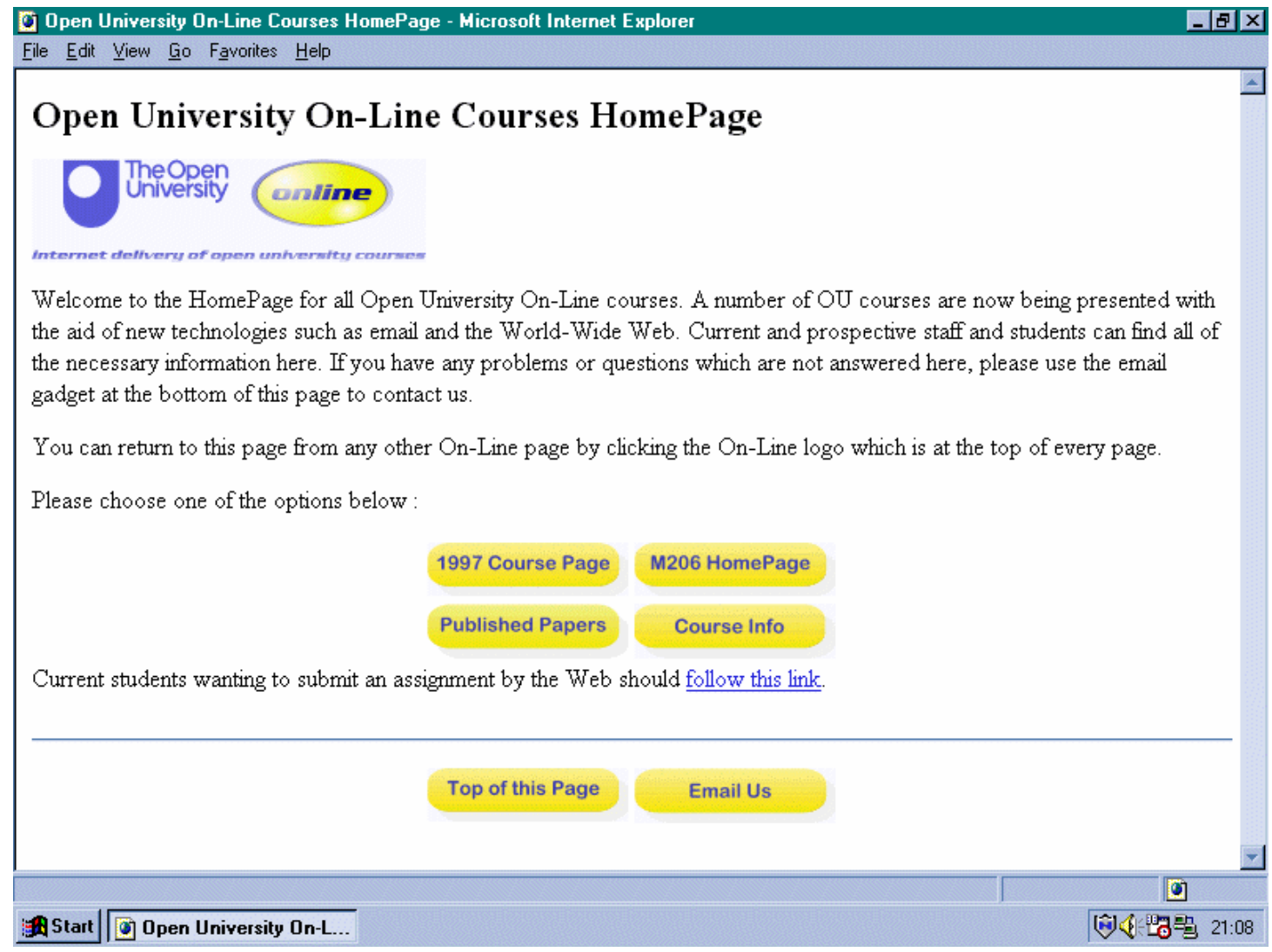

Fig 1: Online Home page

The Web-based reservation/registration system was an attempt both to increase flexibility and speed of access, and to automate data capture. Students can now register using a "smart" Web form (see http://cszx.open.ac.uk) which ensures that mandatory fields are completed, and only valid entries and combinations of entries are submitted. The form results in an offer of registration being e-mailed to the student, which means that there is no bureaucratic delay with incorrectly completed forms, and no postal or administrative delay. In addition, students can register for non-electronic courses and will be sent appropriate printed information via post.

\section{Assignment handling system}

The university has well-established procedures for grading paper Tutor Marked Assignments (hereafter referred to as 'TMAs' in the figures or 'assignments' in the text) which must be interpreted for electronic assignments (See Figure 2). The linchpin is a standard multi-part form which accompanies assignments and accumulates details from student, tutor, administrators and monitor in turn. Students submit assignments to their tutor, who notes grades and comments both on the cover form and on the assignment itself. The assignment is marked in conformance to a scheme specified by the Examination and Assessment Board which also sets the assignments and often provides written 'post-mortem' discussions of them. The tutor then sends the assignment to the central Assignment Handling Office which enters all the information into a database, verifies details, and returns the assignment to the student. 


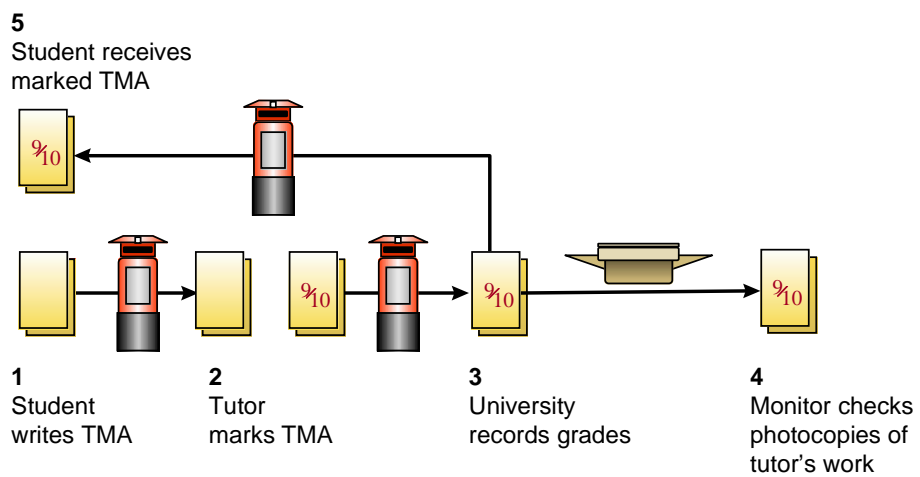

Fig 2 The paper-based assignment handling system

The paper system requires the assignment to be posted three times and potentially to be photocopied at more than one stage (for 'monitoring', part of the quality and staff development procedures). Attempts to speed up this system have introduced additional cost and complexity, making them infeasible at full scale. There are five major disadvantages to the paper system:

i) A student does not know if an assignment has been received;

ii) assignments can get lost, and a student may not have photocopied the work as advised;

iii)The turn-around time reduces the impact of remedial advice;

iv)Completion of the multi-part form is time-consuming and error-prone. If any of the details is incorrect, the tutor is contacted for corrections; this happens in about $5 \%$ of assignments (half a million assignments a year).

v) The variability of communications systems world-wide can make it difficult for students in some countries to study effectively.

The challenge for an electronic assignment (TMA) system was to improve functionality, efficiency, and international access.

The electronic system is Unix-based, involving a centralised collection of databases (see Fig 3), with supporting software written in Perl and Java script. The system is essentially a five-stage process: student assignment submission; tutor assignment receipt; tutor assignment marking; tutor-marked assignment submission and student marked-assignment collection. 


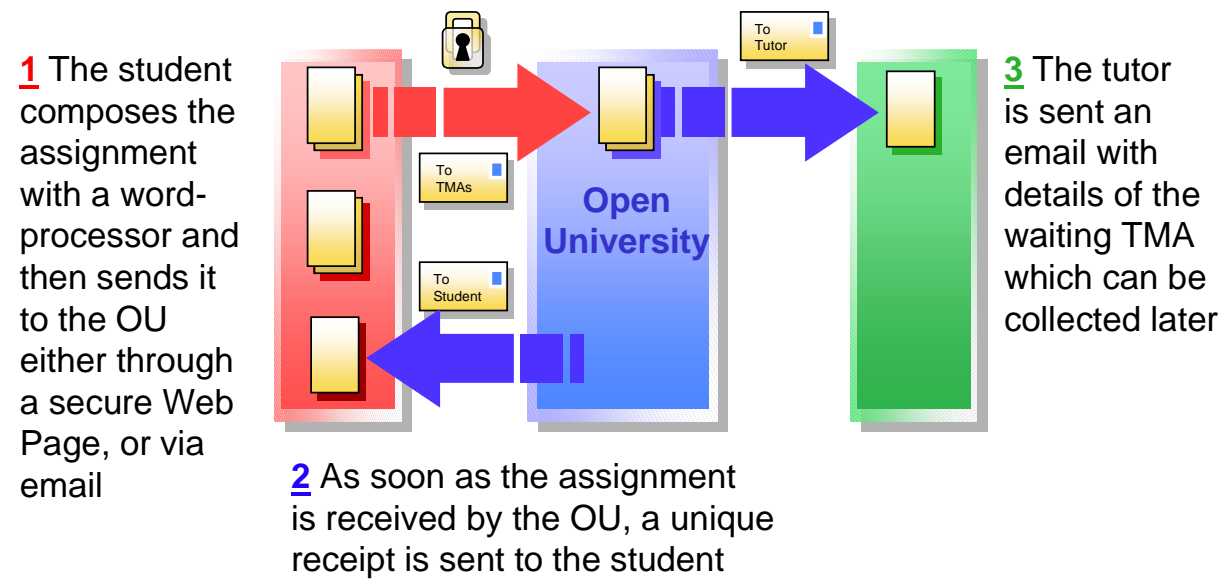

Fig 3 First stage: Student submits an assignment.

\section{Student assignment Submission}

The student submits a word-processed assignment document via a passwordsecured Web form to a central database. The arrival of an assignment immediately causes two reactions:

i. A uniquely-numbered receipt is sent to the student. This audit mechanism is not available in the postal system.

ii. An email message informs the tutor that an assignment awaits downloading.

The multi-part form is automated; the system matches the student's personal identifier (PI) number to the student's details stored in the central database and integrates these into the file sent to the tutor (Figure 3). If the student wishes to submit their assignment 'off-line' then it can be attached to an email message to a special address. In either case the student will usually send the file in a compressed format ('ZIPed') in order to save transmission time.

\section{Tutor assignment Receipt}

The tutor accesses a 'secure' Web page using the tutor's username and password to select waiting unmarked assignments. A copy of the student's assignment and the mark scheme are downloaded automatically onto the tutor's machine in s a single compressed file which minimizes the storage required on the tutor's disk.

\section{Tutor TMA Marking}

The tutor marks and annotates the assignment electronically, using a marking tool (an extension to Microsoft Word 6) which provides various aids to on-screen commenting and grading. The tool performs local validation of marks and computes the overall grade. While seemingly trivial, this facility has eliminated 
the manual error-checking necessary in the paper-based system, in which some 2,000 assignments per week are returned to tutors for correction.

\section{Tutor TMA Submission}

The tutor returns the marked assignment to the university via another passwordprotected Web form and receives a unique receipt number. The details are recorded automatically in the central database (Fig 4).

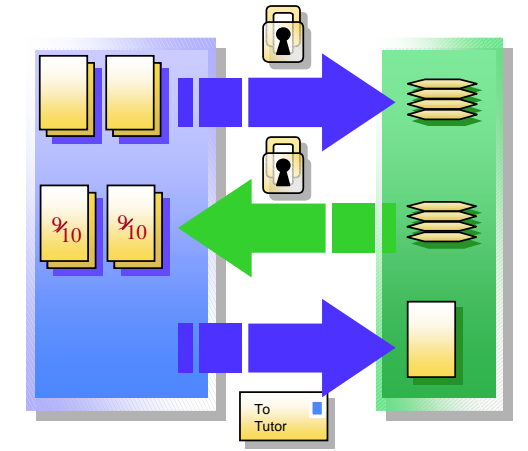

1 The tutor downloads groups of ZIPped TMAs from a secure Web page. The tutor marks the TMAs with a custom-built marking template and then return the marked TMAs as ZIP files to the OU

2 As soon as the assignment is received by the $\mathrm{OU}$, a unique receipt is sent to the tutor by email

Fig 4 Tutor picks up and marks assignments.

\section{Student Collects Marked Assignment}

An email message is despatched automatically from the central computer notifying the student that the marked assignment awaits collection. The student can retrieve the assignment, in compressed (ZIPped) format from a password-secured Web page (Figure 5). 


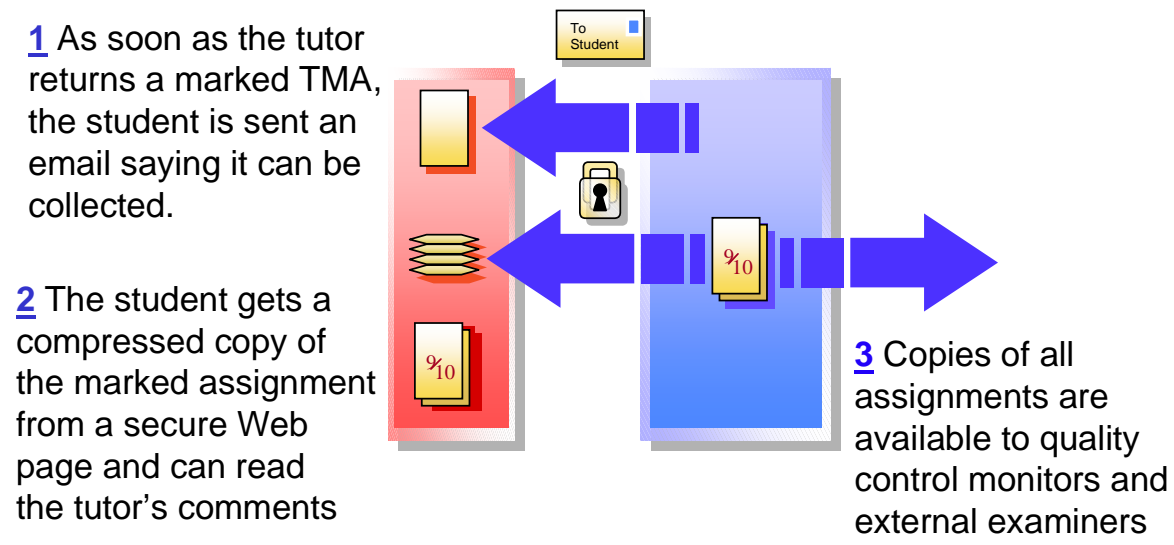

Fig 5 Student collects marked assignment.

The electronic assignment handling system has eliminated some disadvantages of the paper-based system and improved functionality. Student assignment submissions are acknowledged; tutor-marked assignment receipts are acknowledged; copies of marked and unmarked assignments are retained (not possible in the paper-based model); and assignment turn-around time has been substantially reduced (the current record is 4 hours; the typical turnaround time is 5-7 days, compared to 2 weeks for paper).

Improvements stretch beyond the initial goals: administrative processes such as monitoring are facilitated; student progress is easily aggregated and made available to tutors; turn-round time for assignments can be monitored; and photocopying is reduced substantially. The electronic assignment submission system has improved the support given to students and tutors alike; this aspect will be address in more detail in a subsequent paper on the student experience.

\section{Student-tutor interaction}

Focal to the OU's model of supported open learning is the student's interaction with a tutor, who provides academic support to a group of approximately 25 students. In the conventional system, there are two strands to this association: one-to-one queries, and face-to-face tutorials.

\section{One-to-one queries}

Students contact their tutor directly, usually via telephone, to ask questions about course material, assignments, deadlines, OU procedures, or for other general help and support. Most tutors announce hours during which they are available for queries. As students require timely help, the inability to contact a tutor at the crucial moment can result in learning delays and frustration.

\section{Tutorials}

In the broadest characterization, face-to-face tutorials tend to have two main parts: 
1. a diagnostic component, in which instructors clarify

students' progress with respect to coursework, answer questions, and reflect on a previous assignment;

2. a lecture or problem-solving component, in which instructors elicit discussion on examples and issues, or in which students solve and discuss problems.

Instructors are given a 'free hand' in running their tutorials, and they may include any of a variety of activities: open, student-centred, question and discussion sessions; lecture sessions which amplify the course material; worked examples; problemsolving or practical sessions in which students work on problems individually or in small groups. Interaction and discussion are emphasised; tutors see implicit benefits in student-student interaction and peer group assessment.

While such interactions are desirable, experience has shown that many students have difficulty attending face-to-face tutorials. And tutorials are not without their costs: management and maintenance of local study centres; allocation of tutor groups based on locale; organization of local tutorial schedules.

\section{Conferences and Web resources}

The electronic system relies primarily on asynchronous communication through electronic mail and computer conferencing. Our conferencing system, Hypernews, allows one-to-many and many-to-many communication. Conferences are viewed through a Web browser: links to other articles, files and locations on the Web are displayed as icons or hyperlinks. Editing of articles or changing membership information is done through a simple forms-based interface. As course material is moved onto the WWW, tutors will be able to provide answers or present tutorials which refer to course material with live hyperlinks (segments of text which, when selected, link automatically to another, related Web page), rather than static references. The notification facility supports a 'stop press' conference to which all members of the course are automatically subscribed, giving them (a) an automatically-sent e-mail copy of every posting, and (b) read-only access to a reference copy of the announcement. Thus, those students whose access is through a slow Internet connection or simple e-mail are not disadvantaged. Other conferences are available for both social and work-related discussions; most are limited to 20 to 50 students to keep discussions manageable. Students with e-mail only access can treat these conferences as mailing lists and remain full participants.

Tutors have Web access to a database of tutorial material, examples, and frequentlyasked-question sets. A number of tutorial models have been tried and will be reported later in this series, in a paper on the role of the tutor in an electronic environment. All students globally can notionally participate in electronic tutorials: time constraints are reduced when tutorials extend over several days, and location is not an issue.

The conferencing system integrates with existing university systems and automatically generates student-tutor allocations. It also monitors changes so maintenance costs are reduced. Hence, by automating the conference membership and maintenance we have avoided additional administration — potentially a considerable saving. 


\section{Electronic examination}

Assessment is usually based on a combination of assignments and a final closed-book examination normally given in an examination centre under strict supervision. Special arrangements must be made for students outside the UK, which can be quite costly. This is a same time, same place arrangement, with attendant constraints and access problems.

In 1997 we are using the Internet in the examination process. The examination paper is downloaded as an encrypted document via a secure (password protected) Web page by the invigilator (who is given the passwords in advance) in an appointed examination centre. Once the examination has commenced, the student is free to print the examination paper. On completion, the student's examination script (keyed into a word processor) is encrypted and returned via a secure Web page by the invigilator. In this model, it is the invigilator's job to ensure a clean machine (one without files that reference or pertain to course materials) with a network connection. In the examination marking process the electronic examination script will have partiallyautomated grading to assist the examination marker. Details of this will be reported later in this series in the papers on the student experience and the institutional perspective.

To be able to use the Internet to support more of the examination process will require some substantial re-thinking of the assessment model, including a reappraisal of the underlying objectives of written examinations. Research is underway investigating alternative forms of examination that can better exploit the technology. So far, we have established that parts of the process can be effectively managed over the Internet with a resulting reduction in costs.

\section{Continuing work}

The longitudinal study of Internet presentation continues in 1997, with an increased Internet cohort of 550 students and 25 tutors on undergraduate courses. A trial Internet presentation is also being extended to post-graduate courses. In addition, this system is being adopted by the OU's Institute of Educational Technology, which is using it in 1997. This evaluation project has demonstrated the feasibility of such an integrated system, which is being re-implemented by the university and will be a fully operational university service from 1998. This operation will enable the Open University to support the 4,000 students expected to take the new entry-level Computing course in 1998. Subsequently, the University will extend its electronic provision to other disciplines.

Two focal areas for further research are electronic examinations and support for tutors.

electronic examinations: Making effective electronic examinations that escape the constraints of "same time, same place" arrangements requires new examination and delivery techniques. Under particular scrutiny are: the assessment model; suitable assessment techniques; automated examination marking; and examination delivery mechanisms.

support for tutors: One of the advantages of electronic communication is fostering collaboration among a dispersed tutoring staff. Tutors have worked imaginatively and informally; what is needed are Web resources (e.g., a repository of worked examples and 'ideal' discussions) and other tools (e.g., 
fast capture tools for annotating students' work or providing 'canned' demonstrations) to help them construct and share electronic tutorial materials, so that the range of expertise available in the whole staff can be made more generally accessible, and individual experiences can contribute more generally to teaching.

\section{Summary}

The value Internet technology brings to distance education lies not in direct translation from other media but in transformation of support mechanisms to exploit its potential range. Taking a holistic approach to the integration of the electronic tools into the existing administrative infrastructure has proven effective in this case, allowing a better integration of new systems with old than would have been possible piece-meal. Reviewing the whole process from multiple perspectives has paid off, allowing us to provide a relatively simple and economical system which is likely to scale up effectively, although, on the scale of 150,000 students, there is still real concern about managing demands on communications and about consequences of system breakdowns. Supported Internet presentation is not a cheap option, but it may be one that can provide greater flexibility and can shift effort from mundane tasks (administrative details) to teaching. For example, administration is faster and more efficient with electronic assignments. Turnaround time is reduced; less paper is consumed; access to assignments and records is facilitated; and automatic logging increases accountability.

Making a conservative transition to new technology has allowed us to assimilate and evaluate the impact of the technology before deciding on radical culture changes. In fact, making a conservative start may well lead to a more radical transition in the long-run, because, as users - tutors, administrators, students - have been convinced of the feasibility of implementation, they have tended to re-think the fundamental structure of their activities. Users are feeding back to the development team ideas more radical than any the team would have dared to propose. (An evaluation of the Internet presentation from the perspectives of the users will be offered in subsequent papers in this series.)

But the real key to successful application of technology is good teaching: using technology only when it is a cost-effective servant of pedagogy. Experience has shown that it is easy to propose an electronic solution that is more expensive and time-consuming than the paper-based system it is supposed to improve upon (Pilgrim \& Leung, 1996). We must analyse our existing processes holistically, deeply, and critically in order to provide fully- and appropriately-realized Internet teaching that serves learning at least as well, and at least as economically, as conventional methods.

\section{Acknowledgements}

The authors thank the students and tutors who took part in the Internet presentation trials; all the central and regional administrators who helped; David Clover, Adam Freeman, Mike Wright, Barbara Poniatowska, Debra Thompson, Mike Richards, Paula Cole; and the Office for Technology Development. 


\section{References}

Adamson-Macedo, C (1996) PCs, the bandwidth revolution and engineering curricula IEE Computing and Control Engineering Journal 7 (3) 129-135.

Jones, D (1996) Computing by distance education ACM SIGCSE Bulletin 28 (Special Issue) 139-146.

Owen, G S (1996) Integrating World Wide Web technology into undergraduate Education ACM SIGCSE Bulletin 28 Special Issue 101-103.

Pilgrim, C J and Leung Y K (1996) Appropriate use of the Internet in Computer Science Courses ACM SIGCSE Bulletin 28 (Special Issue) 81-86.

Pitt, M (1996) The use of electronic mail in undergraduate teaching British Journal of Educational Technology 27 (1) 45-50.

Reinhardt, A (1995) New Ways to Learn Byte March 51-71

Skillcorn, D B (1996) Using Distributed Hypermedia for Collaborative Learning in Universities Computer Journal 39 (6) 471-482.

Thomas, P G, Carswell, L, Emms, J, Petre, M, Poniatowska, B and Price, B (1996) Distance Education over the Internet ACM SIGCSE Bulletin 28 (Special Issue) 147149. 